\title{
Atividade antibacteriana de fibras têxteis contendo nanopartículas de poli (óxido de etileno-b-ácido lático) com óleos essenciais incorporados
}

\section{Antibacterial activity of textile fibers containing poly(ethylene oxide-b-lactic acid) nanoparticles with incorporated essential oils} \author{
Tatiane Michele Popiolski $^{1,3}$, Markus Wilimzig $^{4}$,
Valdir Soldi
}

\footnotetext{
${ }^{1}$ Departamento de Engenharia e Ciência dos Materiais, Universidade Federal de Santa Catarina, Rua Engenheiro Agronômico Andrei Cristian Ferreira, s/n - Trindade, CEP: 88040-900, Florianópolis, SC, Brasil.

${ }^{2}$ Departamento de Química, Universidade Federal de Santa Catarina, Rua Engenheiro Agronômico Andrei Cristian Ferreira, s/n - Trindade, CEP: 88040-900, Florianópolis, SC, Brasil.

${ }_{3}^{3}$ Departamento de Engenharia, Universidade Federal de Lavras, Av. Dr. Silvio Menicuci, s/n, Aquenta Sol, CEP: $37200-$ 900, Lavras, MG, Brasil.

${ }^{4}$ Instituto Brasileiro de Tecnologia do Couro, Calçado e Artefatos (IBTeC), Rua Araxá, 750, Bairro Ideal, CEP: 93334000, Novo Hamburgo, RS, Brasil.

e-mail: soldi.valdir@gmail.com, markus@ibtec.org.br, tatiane_popiolski@ufla.br
}

\begin{abstract}
RESUMO
Neste estudo foi avaliada a atividade antibacteriana de fibras têxteis contendo nanopartículas de poli(óxido de etileno- $b$-ácido lático) com óleos essenciais incorporados. O sistema desenvolvido demonstrou atividade antibacteriana contra Staphylococcus aureus, sugerindo seu potencial uso como agente antibacteriano. Foram utilizados copolímeros dibloco baseados em um bloco fixo de PEO (5kDa) e dois segmentos de PLA diferentes $(4,5$ e $10 \mathrm{kDa})$. A morfologia, a eficiência do encapsulamento, a interação da blenda de óleos essenciais (incluindo, lavanda, melaleuca, tomilho, cravo, cedro e capim limão) e polímero e a cinética de liberação do agente ativo nas nanopartículas foram avaliadas. $\mathrm{O}$ raio hidrodinâmico das nanopartículas determinado pela dispersão da luz foi afetado pelo tamanho do bloco de poli(ácido lático). A liberação in vitro sugere que a barreira polimérica é capaz de controlar a liberação de óleo. A atividade antibacteriana foi mais eficaz em tecidos com fibras arranjadas aleatoriamente. De acordo com estudos da literatura, nanopartículas obtidas de copolímeros com menor massa molar de PLA favoreceram a liberação da mistura de óleos essenciais. O processo de incorporação dos óleos essenciais nas fibras têxteis mostrou-se eficiente sugerindo viabilidade quanto ao uso desse sistema no controle antibacteriano. Os sistemas desenvolvidos oferecem uma nova estratégia para a liberação controlada com atividade antibacteriana e apresentam potenciais aplicações na indústria calçadista.
\end{abstract}

Palavras-chave: nanopartículas, óleos essenciais, efeito antibacteriano.

\begin{abstract}
In this study was evaluated the antibacterial activity of textile fibers containing poly (ethylene oxide-b-lactic acid) nanoparticles with incorporated essential oils. The developed system demonstrated antibacterial activity against Staphylococcus aureus, suggesting its potential use as antibacterial agent. Diblock copolymers based on a fixed PEO block (5kDa) and two different PLA segments $(4.5$ or $10 \mathrm{kDa})$ were used. The morphology, encapsulation efficiency, blending interaction of essential oils (including lavender, tea tree, thyme, cloves, cedar and lemon grass) and polymer and the release kinetics of the active agent in the nanoparticles. The hydrodynamic radius of the nanoparticles determined by light scattering was affected by the size of the poly(lactic acid) block. The in vitro release suggests that the polymeric barrier is able to control the oil release. Antibacterial activity was more effective in textiles with randomly arranged fibers. In agreement with studies from the literature, nanoparticles obtained from copolymers with lower PLA molar mass favored the release of the essential oil mixture. The incorporation process of the essential oils in the textile fibers proved to be efficient, suggesting viability regarding the use of this system in the antibacterial control. The devel-
\end{abstract}


oped systems offer a new strategy for controlled release with antibacterial activity and suggesting a potential application in the footwear industry.

Keywords: nanoparticles; essential oil; antibacterial effect.

\section{INTRODUÇÃOO}

A utilização quotidiana de materiais têxteis e o consequente contato com pele residual (morta) e suor, representa uma ótima fonte de nutrientes para o desenvolvimento de micro-organismos, entre estes, bactérias e fungos. $\mathrm{O}$ desenvolvimento de bactérias pode, sob condições adequadas de temperatura e umidade, originar maus odores, descoloração, perda de propriedades, deformação e redução do tempo de vida útil das fibras. Além de considerar que Staphylococcus aureus é uma bactéria formadora de toxina que pode comprometer a saúde humana. Portanto, a presença de um agente antibacteriano é não somente uma barreira física, mas também uma barreira química contra a ação de agentes biológicos indesejáveis [1], pois de maneira geral, a simples lavagem dos tecidos não é suficiente para eliminar estes microrganismos, ocorrendo somente uma redução destes, sendo que os sobreviventes rapidamente se multiplicam a cada nova utilização. A característica estrutural das fibras têxteis, associada a ambientes de alta umidade e temperatura, proporciona um ambiente favorável para o surgimento e crescimento de microrganismos responsáveis pelo mofo, bolores e manchas. [2-3] O controle de microrganismos em materiais têxteis se estende a diversas áreas como ambientes hospitalares, fitness, equipamentos militares, produtos de cama, mesa e banho, vestuário de uso diário e em materiais utilizados em calçados, que é o foco do presente estudo. Por longo tempo, diferentes aditivos químicos foram utilizados para tratamento antibacteriano em materiais têxteis, incluindo desde substâncias simples como íons de halogênios até os mais complexos como detergentes especiais. [3] Atualmente os compostos organoclorados e derivados de arsênio vem sendo substituídos por materiais nanoestruturados sintetizados a partir de sais metálicos, como óxido de zinco, com propriedades antibacterianas. [4]

Devido ao vasto campo de aplicação de sistemas nanoestruturados, novas propriedades que não são encontradas em escala micro e macro, podem ser observadas em escala nano, como tolerância à temperatura, variedade de cores, alterações na reatividade química e condutividade elétrica. [5] Mais recentemente, o uso da nanotecnologia tem favorecido o desenvolvimento de soluções que incluem a incorporação de nanoestruturas (p.ex. nanopartículas) em fibras têxteis pelo método de adsorção. Este processo consiste em tratar o substrato (material têxtil) com nanoestruturas sob condições controladas. [6] Por exemplo, BALASUBRAMANIAN et al. [7] desenvolveram nanofibras de poliacrilonitrila (PAN) com óleo essencial de lavanda pelo método eletrospinnig. $\mathrm{O}$ foco da pesquisa realizada pelos autores foi a aplicação do sistema nanoestruturado como um material antibacteriano de liberação controlada, na área biomédica, setor têxtil e no tratamento de águas. [8] Pesquisadores da Universidade de Cornell-USA desenvolveram roupas High-tech com propriedades antibacterianas e autolimpantes. A estratégia consistiu na aplicação de nanopartículas de prata e/ou de paládio em tecidos de algodão. Além do efeito antibacteriano, a prata incorporada à fibra de algodão aumentou a periodicidade de lavagem das fibras, evitando dessa forma a sujidade e a formação de manchas devido ao uso frequente. [8] A incorporação de nanopartículas de ouro em matrizes poliméricas formadas por poliestireno e poli(dimetilsiloxano) pelo método de imersão em solução foi avaliada por CORBIERRE et al. [9] Neste caso específico os autores utilizaram um revestimento nas nanopartículas compatível com a matriz polimérica evitando assim, a formação de agregados em solução. Os estudos de incorporação e liberação das nanopartículas da matriz polimérica foram realizados via espectroscopia de UV-Visível, enquanto a morfologia e tamanho das nanopartículas foram analisadas por microscopia eletrônica de transmissão. [10] O mesmo sistema nanoestruturado foi também avaliado pelos autores considerando a preparação prévia das nanopartículas de ouro e a incorporação destas em matrizes de poliestireno e poli(dimetilsiloxano) via polimerização in situ. Os resultados mostraram uma dispersão uniforme das nanopartículas na matriz polimérica. [10, 11] Em outro exemplo, pesquisadores do Departamento de Agricultura dos Estados Unidos (USDA) produziram nanopartículas de prata diretamente sobre fibras de algodão utilizando água e poli(etileno glicol) (PEG), com o objetivo de reduzir o mau odor de tecidos, como consequência da redução do crescimento e proliferação de microrganismos. [10] Com o mesmo objetivo, BALAKUMARAN et al. [11] trataram tecidos de algodão com nanopartículas de prata, sendo que os resultados apresentaram excelente atividade antibacteriana contra todos os testes patogênicos. A redução de mais de $93 \%$ do número de colônias bacterianas foi registrada contra todos os patógenos testados.

Sistemas nanoestruturados dessa natureza têm sido utilizados em calçados, particularmente, em palmilhas, forros internos, solados de base polimérica e cabedal, reduzindo dessa forma o crescimento e a proliferação de bactérias responsáveis pela formação do mau cheiro. Empresas como a REINSTE NANO VENTURES [12] da Índia em parceria com a SILANOTEX [13] da Alemanha vêm trabalhando com a incorporação de nanopartículas de prata em fibras sintéticas como as utilizadas em componentes do calçado, com o objetivo de evitar a proliferação de germes patogênicos. Neste contexto, a indústria calçadista busca se modernizar e atualizar suas tecnologias e aplicações, tanto no desenvolvimento e utilização de materiais antibacterianos, 
como no desenvolvimento de dispositivos viáveis para evitar o acúmulo de umidade no interior do calçado. Por exemplo, as empresas Reebok e Ferracini desenvolveram a tecnologia Play Dry [14, 15] que, através da combinação dos processos de dispersão e canalização, acelera a evaporação da umidade produzida pelo corpo humano. Para a dispersão são utilizadas fibras hidrofílicas na parte interna para atrair a umidade e fibras hidrofóbicas que dispersam o suor acelerando a evaporação. E a canalização utiliza fibras cruzadas como meio de saída para a umidade, permitindo uma evaporação mais rápida. A empresa Dublauto Gaúcha desenvolveu uma palmilha com tratamento nanotecnológico antibacteriano, que tanto previne o mau cheiro, como também algumas doenças alérgicas para o usuário. A mesma empresa desenvolveu também o forro Dry Soft que absorve rapidamente o suor dos pés, evitando que este tenha contato com a pele. Ao mesmo tempo, microcápsulas com fragrâncias impregnadas no forro são liberadas continuamente, devido ao atrito natural no caminhar.

Neste caso específico, as microcápsulas além de evitar o mau cheiro, agem também como hidratante da pele, auxiliando na cicatrização de pequenas fissuras, na regeneração das células e na ativação da circulação sanguínea. [16]

O processo de incorporação de nanopartículas em fibras têxteis, como ilustrado na Figura 1, protege os ativos da oxidação e volatização, proporcionando sensações e fragrâncias no momento desejado através do sistema de liberação gradativo que é acionado pela fricção e atrito provocado pelo uso diário.

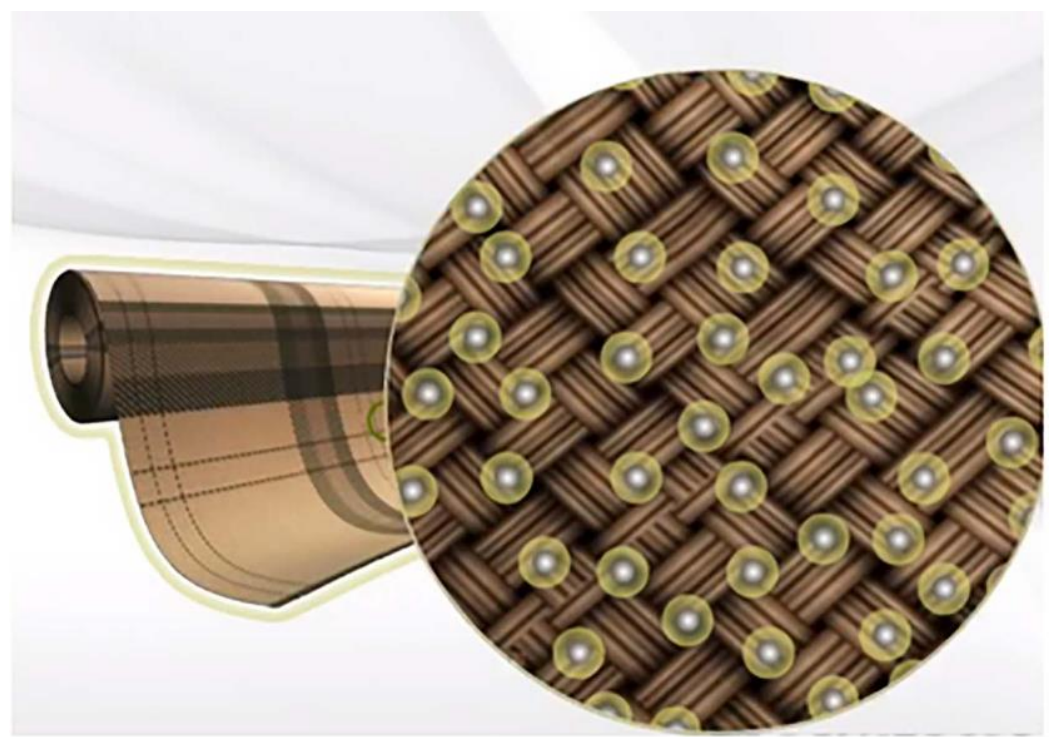

Figura 1: Esquema ilustrativo de têxtil contendo nanopartículas. [17]

Neste aspecto, a nanotecnologia tem um papel preponderante tanto na utilização, como na eficiência de agentes antibacterianos que tem a função de inibir a transferência e o alastramento do contato com os microrganismos patogênicos e, como consequência, reduzir o odor causado pela degradação microbiana e evitar a perda das características das fibras. [18] Alguns dos antibacterianos mais eficientes usados atualmente foram desenvolvidos paralelamente ao avanço da nanotecnologia pois levam em sua composição, nanopartículas. [19]

Este estudo teve como objetivo principal avaliar a atividade antibacteriana de fibras têxteis contendo nanopartículas de poli(óxido de etileno- $b$-ácido lático) com óleos essenciais incorporados. Técnicas de espalhamento de luz e microscopia eletrônica de transmissão foram utilizadas para caracterizar os sistemas nanoestruturados. Os estudos de liberação de óleos essenciais das nanopartículas e destas incorporados em fibras têxteis foram avaliados por espectroscopia UV-Visível.

\section{MATERIAIS E MÉTODOS}

\subsection{Materiais}

Foram utilizadas nanopartículas pré-sintetizadas a partir do copolímero dibloco poli(óxido de etileno)- $b$ poli(ácido lático), fornecido pela PolymerSourceTM, com a presença de uma blenda de óleos essenciais (incluindo, lavanda, melaleuca, tomilho, cravo, cedro e capim limão) fornecida pela empresa Nanovetores de Florianópolis, SC. Foram utilizadas dois copolímeros de diferentes massas molares: PEO5 $\mathrm{kDa}_{-}-b$ PLA $_{4,5 \mathrm{kDa}} \mathrm{e}$ 
$\mathrm{PEO}_{5 \mathrm{kDa}}-b-\mathrm{PLA}_{10 \mathrm{kDa}}$. A metodologia para a preparação das nanopartículas foi a mesma utilizada por POPIOLSKI et al. [20] Como solvente foi utilizado Acetona P.A fornecida pelo Group Carlo Erba Reagents e água ultra-pura produzida pelo equipamento Milli-Q Billerica, MA, EUA. Como materiais têxteis de estudo foi utilizado um tecido de sarja branca $100 \%$ algodão, utilizado como forro em calçados esportivos e em calçados funcionais. Como não tecido, foi utilizado um material $100 \%$ poliéster empregado como base para laminados sintéticos e reforço em calçados, ambos fornecidos pela empresa Dublauto Gaúcha de Novo Hamburgo-RS.

\subsection{Preparação das nanopartículas}

A técnica consiste na dissolução do copolímero em um solvente termodinamicamente bom para os dois blocos, ou seja, compatível para os dois blocos, favorecendo a dissolução do copolímero como um todo, em nosso caso, a acetona, sob agitação constante, a temperatura ambiente, seguida de adição lenta e progressiva de um solvente seletivo para um dos blocos (água ultra-pura), [21] onde o bloco solvofóbico se organiza minimizando o contato com o solvente, favorecendo assim, dependendo das condições experimentais, a formação de nanoestruturas bem definidas em escala nanométrica. [22] A taxa de adição do solvente seletivo e a velocidade de agitação magnética foram cuidadosamente estabelecidas após diversos experimentos, a fim de promover a formação de estruturas bem definidas, homogêneas, na ausência de agregados, ou seja, com um baixo índice de polidispersidade. [23] Mais especificamente, o copolímero na concentração de $40 \mathrm{mg} \mathrm{mL}^{-1} \mathrm{e}$ a blenda de óleos essenciais (10\%) foram dissolvidos em $2 \mathrm{~mL}$ de acetona. Esta solução foi então gotejada a uma taxa constante de $7,63 \mathrm{~mL} \mathrm{~h}^{-1} \mathrm{em} 4 \mathrm{~mL}$ da solução aquosa com o uso de uma seringa de $2 \mathrm{~mL}$ acoplada à uma bomba de infusão, sob agitação magnética de $2500 \mathrm{rpm}$. A eliminação do solvente orgânico foi realizada por evaporação sob pressão reduzida em um rotavapor da marca Janke\&Kunkel IKA Labortechnik. Neste processo de formação de nanopartículas em água, o bloco hidrofílico (PEO) é orientado para a formação do revestimento das nanopartículas (camada externa) enquanto que a parte hidrofóbica, o PLA, é protegida do meio externo, formando o núcleo. O núcleo hidrofóbico atua como um micro ambiente ideal para a encapsulação do agente ativo (óleo essencial), também de caráter hidrofóbico, enquanto que o bloco em contato com o solvente forma uma interface estabilizante entre o núcleo e o meio externo. Para evitar a aglomeração das nanopartículas, todas as amostras foram diluídas a $10 \mathrm{~mL}$.

\subsection{Caracterização das nanopartículas}

\subsubsection{Espalhamento de luz estático e dinâmico}

No Espalhamento de Luz Estático (SLS), a intensidade da luz espalhada é coletada em função do ângulo de espalhamento, enquanto no Dinâmico (DLS), a correlação da intensidade da luz espalhada é obtida em função do tempo, para um dado ângulo fixo. As duas análises fornecem informações complementares a respeito dos sistemas estudados. Particularmente neste estudo, a técnica de DLS foi utilizada para analisar a forma, número de agregação e as interações das nanopartículas e correlacioná-las com as dimensões das mesmas em solução aquosa, através de flutuações temporais na luz espalhada que geram informações a respeito da dinâmica das partículas em solução. [23]

As análises foram realizadas utilizando um goniômetro ALV/CG6-8F equipado com um laser de hélionéon vermelho $(\lambda=632,8 \mathrm{~nm}$ ), operando a uma potência de $35 \mathrm{~mW}$ e um dispositivo de correlação digital ALV/LSE-5004, com um tempo de amostragem inicial de $125 \mathrm{nS}$. O ângulo de dispersão acessível do equipamento varia de 12 a $155^{\circ}$. Para esta análise, as amostras foram filtradas com filtro de acetato de celulose de $0,45 \mu \mathrm{m}$, e medidas em células cilíndricas de $10 \mathrm{~mm}$ de diâmetro, imersas em um banho de tolueno com temperatura regulada a $25{ }^{\circ} \mathrm{C}$. O módulo de dispersão (q) é definido pela equação 1 , na qual $\eta$ representa o índice de refração de água pura, $\theta$ o ângulo de dispersão e $\lambda$ o comprimento de onda.

$$
q=\frac{4 \pi \eta}{\lambda} \operatorname{sen}\left(\frac{\theta}{2}\right)
$$

As amostras foram sistematicamente estudadas a $90^{\circ}, 60^{\circ}$ e $120^{\circ}$ e cada experimento foi realizado durante 300s. As amostras também foram estudadas em varredura de diferentes ângulos de espalhamento, variando de $40^{\circ}$ até $140^{\circ} \mathrm{com}$ aumento escalonado de $10^{\circ}$. A intensidade de espalhamento foi corrigida considerando as contribuições do solvente (água) e o tolueno (padrão), bem como a alteração do volume de dispersão, com o ângulo de detecção. As distribuições de tempos de relaxação, $\mathrm{A}(\mathrm{t})$, foram obtidos por meio do método CONTIN [24, 25] aplicadas às funções de autocorrelação, $C(q, t)$. [26] $O$ raio hidrodinâmico $\left(R_{H}\right)$ foi determinado usando a equação de Stokes-Einstein (equação 2), onde $\mathrm{k}_{\mathrm{B}}$ é a constante de Boltzmann (em J K ${ }^{-1}$ ), $\mathrm{T}^{\mathrm{e}}$ a temperatura (em K), D é o coeficiente de difusão e $\eta$ é a viscosidade do meio (água pura), neste caso, $(\mathrm{g}=$ $0,89 \mathrm{cP}$ a $\left.25^{\circ} \mathrm{C}\right)$. 


$$
R_{H}=\frac{K_{B} T}{6 \pi \mathrm{\eta} D}
$$

O raio de giro $(\mathrm{Rg})$ foi calculado a partir da parte elástica (I (q)), da intensidade de dispersão utilizando a aproximação de Guinier (equação 3).

$$
\ln l=\ln I_{0}-\frac{1}{3} q^{2} R g^{2}
$$

A relação entre $\mathrm{Rg}$, obtido a partir dos dados de SLS aplicando a equação de Guinier, e $\mathrm{R}_{\mathrm{H}}$, obtido via DLS e a equação de Stokes-Einstein, fornece o grau de anisotropia $(\rho)$.

\subsubsection{Microscopia eletrônica de transmissão}

A morfologia das nanopartículas foi avaliada através da microscopia eletrônica de transmissão (MET) em um microscópio CM200 Philips (FEI Company, Hillsboro, EUA) operado a $80 \mathrm{kV}$, com a finalidade de verificar a morfologia e a estrutura das nanopartículas. As amostras foram diluídas 10 vezes em água ultra-pura e foram depositados $7 \mu \mathrm{L}$ de amostra sobre grids de cobre revestidos com carbono e antes da secagem completa adicionou-se $7 \mu \mathrm{L}$ da solução de acetato de uranila $2 \%(\mathrm{w} / \mathrm{v})$ corando negativamente as amostras. Depois de alguns minutos, o excesso de líquido foi retirado com papel filtro e a grid foi deixada para secar. As imagens foram gravadas em filmes Kodak SO163, e os negativos foram digitalizados off-line usando uma câmera Kodak Mega Plus CCD. As micrografias obtidas foram tratadas no software Image Tool for Windows para analisar o diâmetro das nanopartículas, contando com cerca de 400-500 contagens por amostra.

\subsection{Incorporação das nanopartículas em fibras têxteis}

Para este estudo foram utilizados dois tipos de fibras têxteis, uma sarja branca $100 \%$ algodão, identificada como tecido A, e um não tecido composto com $100 \%$ poliéster, como tecido B. As nanopartículas contendo a mistura de óleos essenciais foram adsorvidas aos têxteis por imersão dos tecidos em solução contendo as nanopartículas.

\subsection{Perfil de liberação dos óleos essenciais}

Para análise do perfil de liberação dos óleos encapsulados às nanopartículas adsorvidas aos têxteis, amostras com $1,0 \mathrm{~cm}^{2}$ de área foram inicialmente mantidas em estufa a $37{ }^{\circ} \mathrm{C}\left( \pm 2{ }^{\circ} \mathrm{C}\right)$, durante 58 dias. Após este período, cada amostra avaliada foi embebida em $1 \mathrm{~mL}$ de solvente orgânico (acetona) e após 10 minutos de ultrasonificação, as soluções de lavagem foram avaliadas por Espectroscopia de UV-Visível, a 210 e $220 \mathrm{~nm}$, [27, 28] em um espectrofotômetro de feixe duplo, modelo CARY 5000 - Varian. Todas as amostras analisadas por espectroscopia foram diluídas em acetona.

\subsection{Atividade antibacteriana}

Conforme a norma AATCC 100-2004, [29] foram utilizados corpos de prova circulares de 4,8 $\pm 0,1 \mathrm{~cm}$ de diâmetro, suficientes para absorver $1,0 \pm 0,1 \mathrm{~mL}$ do inóculo. Para alcançar esta condição, dois corpos de prova foram necessários. Todos os têxteis foram previamente esterilizados em autoclave. Os corpos de prova foram inoculados com Staphylococcus aureus, cepa ATCC 6538, organismo gram positivo. Os resultados foram expressos em percentuais de redução do número de bactérias no inóculo após $24 \mathrm{~h}$ de contato com as fibras têxteis. Estas análises foram realizadas no Laboratório de Microbiologia do Instituto Brasileiro de Tecnologia do Couro, Calçado e Artefatos (IBTeC) de Novo Hamburgo-RS.

\section{RESULTADOS E DISCUSSÃO}

\subsection{Caracterização das nanopartículas}

\subsubsection{Espalhamento de luz dinâmico}

Para determinar a distribuição de tamanho e grau de polidispersidade, estudamos a dependência angular da intensidade de espalhamento de luz das soluções de nanopartículas de PEO- $b$-PLA. Uma vez que as nanopartículas em solução estão em constante movimento, as flutuações da intensidade da luz espalhada estão diretamente relacionadas ao movimento browniano. Portanto a técnica de Espalhamento de Luz Dinâmico (DLS) foi utilizada para analisar este movimento e correlacioná-lo com as dimensões das nanopartículas em solução 
aquosa, através de flutuações temporais na luz espalhada que geram informações a respeito da dinâmica das partículas em solução. [25]

A Figura 2 mostra a função de autocorrelação C(q, t), calculada utilizando a análise CONTIN, para as amostras medidas nos ângulos de $60^{\circ}, 90^{\circ}$ e $120^{\circ}$ de espalhamento e distribuição dos tempos de relaxação medidos no ângulo de $90^{\circ}$. A distribuição do tempo de relaxação obtido a partir de PEO- $b$-PLA automontados em diferentes massas molares de copolímero mostrou a presença de duas populações micelares para as amostras sem o ativo encapsulado (Figuras 2A), onde a primeira corresponde a pequenas micelas enquanto que a segunda população corresponde provavelmente as vesículas ou agregados micelares irregulares, formados por interações dinâmicas entre as cadeias do copolímero. A natureza dinâmica de tais interações foi confirmada, uma vez que não foram detectados os agregados micelares após a filtração em filtro de acetato de celulose $(0,45 \mu \mathrm{m})$.
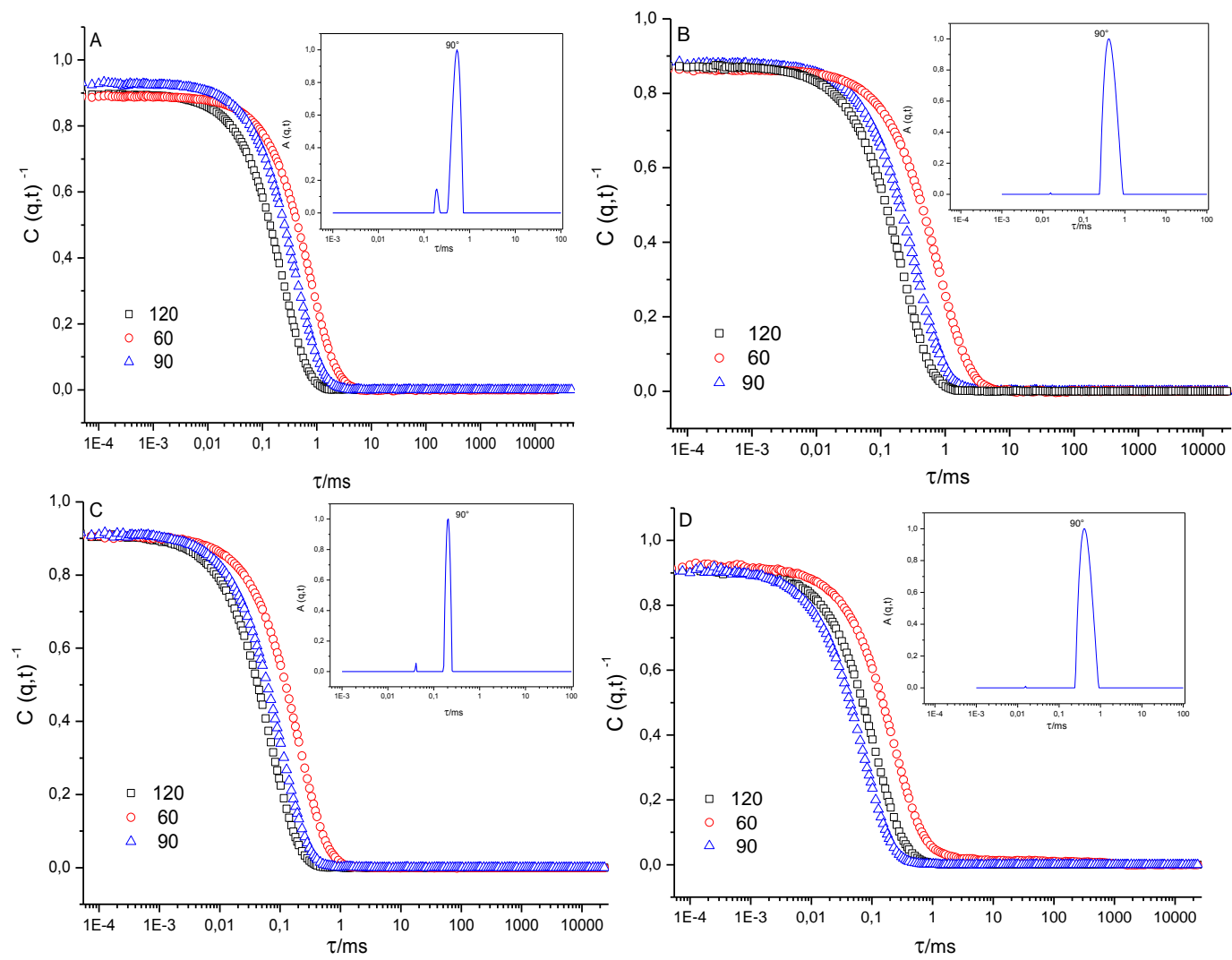

Figura 2: Função de correlação medida por DLS a $90^{\circ}, 60^{\circ}$ e $120^{\circ}$ e distribuição dos tempos de relaxação obtido num ângulo de $90^{\circ}$ para: $\mathrm{PEO}_{5 \mathrm{kDa}}-b-\mathrm{PLA}_{4,5 \mathrm{kDa}}(\mathrm{A})$; $\mathrm{PEO}_{5 \mathrm{kDa}}-b-\mathrm{PLA}_{4,5 \mathrm{kDa}}$ com a Blenda (B); $\mathrm{PEO}_{5 \mathrm{kDa}}-b-\mathrm{PLA}_{10 \mathrm{kDa}}$ (C); $\mathrm{PEO}_{5 \mathrm{kDa}}-b-\mathrm{PLA}_{10 \mathrm{kDa}}$ com a Blenda (D).

O raio hidrodinâmico $\left(\mathrm{R}_{\mathrm{H}}\right)$ foi obtido através do gráfico de $\Gamma$ versus $\mathrm{q}^{2}$ calculado usando a relação de Stokes-Einstein (Equação 2). Os gráficos indicam um comportamento difusivo do espalhamento das partículas e a existência de objetos esféricos para os dois sistemas estudados, micelas sem ativo encapsulado ou com a presença deste.

Os resultados de DLS indicam que ocorreu um aumento no tamanho das nanopartículas com a presença do ativo encapsulado e uma distribuição bimodal para os copolímeros sem a presença deste. As nanopartículas sem óleo essencial do sistema de $\mathrm{PEO}_{5 \mathrm{kDa}}-b-\mathrm{PLA}_{4,5 \mathrm{kDa}}$ (Figura 2A) tem raios hidrodinâmicos de $77 \mathrm{~nm}$ e $13 \mathrm{~nm}$ para as duas populações observadas. Na presença de $10 \%$ da blenda de óleos essenciais (BD), (Figura 2B) o raio hidrodinâmico aumentou para $92 \mathrm{~nm}$, confirmando a influência do componente oleoso sobre o diâmetro da partícula, assim como reportado por SCHAFFAZICK et al. [30] Para o sistema $\mathrm{PEO}_{5 \mathrm{kDa}}-b$ $\mathrm{PLA}_{10 \mathrm{kDa}}$, que também apresentou duas populações para o sistema vazio, os valores foram $19 \mathrm{~nm}$ e $4 \mathrm{~nm}$ na ausência do óleo (Figura 2C), aumentando para 22,7 nm com 10\% da BD (Figura 2D). 


\subsubsection{Espalhamento de luz estático}

Para os sistemas em investigação os valores de raio de giro $(\mathrm{Rg})$ foram determinados a partir de um gráfico de $\ln$ I versus $\mathrm{q}^{2}$. A Figura 3 apresenta os gráficos da dependência da frequência de relaxação $(1 / \tau)$ do quadrado do modulo do vector de onda $\left(\mathrm{q}^{2}\right)$ medido nos ângulos de 40 até $140^{\circ}$ para as amostras de $\mathrm{PEO}_{5 \mathrm{kDa}}-b$ $\mathrm{PLA}_{4,5 \mathrm{kDa}}$ e $\mathrm{PEO}_{5 \mathrm{kDa}}-b-\mathrm{PLA}_{10 \mathrm{kDa}}$ após filtração em acetato de celulose $0,45 \mu \mathrm{m}$, cujo valor foi calculado com base na lei de Guinier, a partir do coeficiente angular da reta utilizando a Equação 3. Esta aproximação é bastante utilizada para a determinação de $\operatorname{Rg}$ de partículas, sendo válida apenas quando $\mathrm{qRg}<1$.

A razão entre $\mathrm{Rg}$ e $\mathrm{R}_{\mathrm{H}}$ fornece o grau de anisotropia $(\rho)$, que estima a morfologia das nanopartículas. A Tabela 1 mostra os valores de $\rho$ calculados teoricamente para as morfologias mais frequentemente encontradas.

Tabela 1: Valores de $\rho$ e a morfologia das partículas.

\begin{tabular}{c|c}
\hline MORFOLOGIA & $\boldsymbol{\rho}$ \\
\hline Esfera homogênea (hard) & 0,778 \\
\hline Esfera (soft) & 0,977 \\
\hline Vesícula & $>1,00$ \\
\hline Polímero enovelado monodisperso & $1,50 \mathrm{a} 1,70$ \\
\hline Polímero enovelado polidisperso & $>1,70$ \\
\hline
\end{tabular}

Fonte: (adaptado de Burchard). [31]
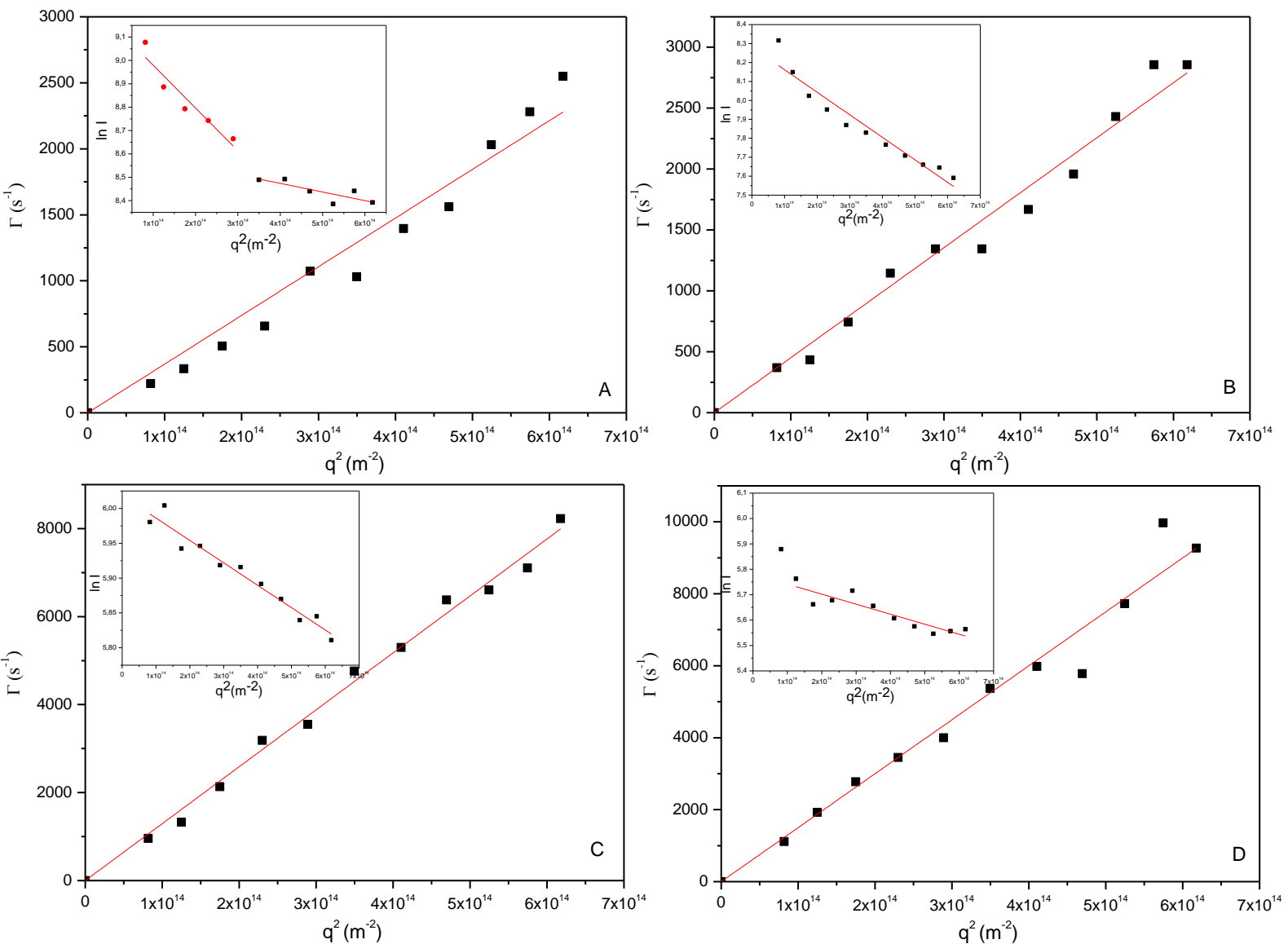

Figura 3: Dependência da frequência de relaxação $(1 / \tau)$ do quadrado do modulo do vector de onda $\left(\mathrm{q}^{2}\right)$ e gráficos de $\ln \mathrm{I}$ versus quadrado do modulo do vector de onda $\left(\mathrm{q}^{2}\right)$ das soluções aquosas de $\mathrm{PEO}_{5 \mathrm{kDa}}-b-\mathrm{PLA}_{4,5 \mathrm{kDa}}(\mathrm{A}) ; \mathrm{PEO}_{5 \mathrm{kDa}}-b-$ $\mathrm{PLA}_{4,5 \mathrm{kDa}}$ com a BD (B); $\mathrm{PEO}_{5 \mathrm{kDa}}-b-\mathrm{PLA}_{10 \mathrm{kDa}}(\mathrm{C}) ; \mathrm{PEO}_{5 \mathrm{kDa}}-b-\mathrm{PLA}_{10 \mathrm{kDa}}$ com a BD (D).

Na Figura 3A são observadas duas inclinações para as amostras de $\mathrm{PEO}_{5 \mathrm{kDa}}-b$ - $\mathrm{PLA}_{4,5 \mathrm{kDa}}$, correspondentes aos valores de $\mathrm{Rg}$ de $74 \mathrm{~nm}$ e $43 \mathrm{~nm}$, respectivamente. Considerando o valor de $\mathrm{R}_{\mathrm{H}}$ de $62 \mathrm{~nm}$ (DLS), 
foram obtidos valores do grau de anisotropia $(\rho)$ de 1,19 e 0,70, respectivamente, que estão relacionadas a morfologia de vesículas e esferas homogêneas. [33] Para as amostras $\mathrm{PEO}_{5 \mathrm{kDa}}-b-\mathrm{PLA}_{4,5 \mathrm{kDa}}$ na presença da blenda de óleos (Figura 3B), o Rg foi de $54 \mathrm{~nm}$, definindo um grau de anisotropia de 0,72 associado a formação de esferas homogêneas.

A partir da Figura $3 \mathrm{C}$ observa-se uma dependência linear para as amostras de $\mathrm{PEO}_{5 \mathrm{kDa}}-b$-PLA $10 \mathrm{kDa}$, correspondente ao valor de $\mathrm{Rg}$ de $20 \mathrm{~nm}$, que corrobora com o valor de $\mathrm{R}_{\mathrm{H}}$ de $17 \mathrm{~nm}$ determinado por DLS. A partir da razão entre Rg (SLS) e $R_{H}$ (DLS) é determinado o grau de anisotropia ( $\rho$ ), que neste caso corresponde ao valor de 1,17 .

$\mathrm{Na}$ presença da blenda de óleos (Figura 3D), obteve-se um $\mathrm{Rg}$ de $16 \mathrm{~nm}$, que resultou num grau de anisotropia de 0,80 . As amostras com $\mathrm{PEO}_{5 \mathrm{kDa}}$-b-PLA $\mathrm{PLk}_{10 \mathrm{kD}}$, nas mesmas condições experimentais, apresentaram comportamento semelhante ao observado por microscopia eletrônica de transmissão (MET). Estes resultados são apresentados na seção 3.1.3, e diferem, no entanto, dos resultados obtidos por SLS, que sugerem a formação de vesículas ou até mesmo um enovelamento polimérico em solução, conforme apresentado na Tabela 1.

Com base na dependência da frequência de relaxação $(1 / \tau)$ do quadrado do modulo do vector de onda $\left(\mathrm{q}^{2}\right)$ medido nos ângulos de 40 até $140^{\circ}$ (Figura 3), foi calculado o raio hidrodinâmico real para os dois sistemas estudados (ver Tabela 2).

Tabela 2: Raio hidrodinâmico real determinado pela dependência angular por SLS.

\begin{tabular}{l|c|c}
\hline \multirow{2}{*}{ COPOLÍMERO } & \multicolumn{2}{|c}{$\mathbf{R}_{\mathbf{H}}$} \\
\cline { 2 - 3 } & NP SEM ÓLEO & NP COM 10\% DE ÓLEO \\
\hline $\mathrm{PEO}_{5 \mathrm{kDa}}-b-\mathrm{PLA}_{4,5 \mathrm{kDa}}$ & $61,30 \mathrm{~nm}$ & $74,80 \mathrm{~nm}$ \\
\hline $\mathrm{PEO}_{5 \mathrm{kDa}}-b-\mathrm{PLA}_{10 \mathrm{kDa}}$ & $16,50 \mathrm{~nm}$ & $19,70 \mathrm{~nm}$ \\
\hline
\end{tabular}

Pelos resultados apresentados, podemos observar aumento no tamanho das nanopartículas com a presença do agente ativo. Esse comportamento é um indicativo de que o processo de encapsulação foi eficiente. Ainda, nas amostras com a presença do agente ativo é observada uma leve dependência angular no espalhamento da solução, que pode ser indicativo da presença de partículas grandes, ou seja, de um sistema polidisperso.

O aumento do teor de PLA na composição do copolímero gera uma diminuição do tamanho de partícula. Podemos considerar duas possibilidades para justificar esta situação: (i) formam-se pequenas nanopartículas que se agregam para formar aglomerados, como reportado por XU [32] para copolímeros de PS- $b$-PEO (poli(óxido de etileno)-estireno). No caso do $\mathrm{PEO}_{5 \mathrm{~K}}-b$-PLA $\mathrm{A}_{4.5 \mathrm{~K}}$, a presença de uma proporção maior de PEO pode favorecer a formação de agregados termodinamicamente reversíveis em solução aquosa. Esta formação está associada a presença de um conjunto de cadeias de PEO na camada externa das nanopartículas. A tendência observada nos resultados obtidos por DLS está de acordo com a conclusão de XU [34] de que o grau de associação das micelas aumenta com o aumento da massa molar do copolímero ou com o aumento o teor hidrofóbico deste, em nosso caso, aumento da proporção de PLA. [34]

\subsubsection{Microscopia eletrônica de transmissão}

A análise por microscopia eletrônica de transmissão (MET) foi realizada com o objetivo de confirmar o tamanho das nanopartículas, uma vez que de acordo com a literatura é aconselhável que se utilize mais de um tipo de metodologia para a determinação dos mesmos, [33] bem como analisar a morfologia das nanopartículas de PEO- $b$-PLA. Conforme observado na Figura 4, para ambos os sistemas estudados foram obtidas nanopartículas esféricas. Comportamento semelhante foi descrito por JIE et al. [34] e NECKEL E SENNA [35] para os sistemas PEO-PLA e PLA-PEG, respectivamente.

As nanopartículas obtidas com o copolímero $\mathrm{PEO}_{5 \mathrm{kDa}}-b-\mathrm{PLA}_{4,5 \mathrm{kDa}}$, observadas na Figura 4 , mostraram diâmetros médios de $38 \mathrm{~nm}$ para micelas sem óleo (4A), respectivamente. Na condição de menor massa de PLA (Figura 4A e 4B), observou-se grandes vazios sobre as grids indicando que a solução de $\mathrm{PEO}_{5 \mathrm{kDa}}-b$ $\mathrm{PLA}_{4,5 \mathrm{kDa}}$ não cobriu a totalidade da superfície das grids, favorecendo a polidispersão. Com o aumento da massa molar de PLA, $\left(\mathrm{PEO}_{5 \mathrm{kDa}}-b-\mathrm{PLA}_{10 \mathrm{kDa}}\right)$, a solução cobriu uniformemente toda a superfície das grids, como observado na Figura 4C e 4D.

$\mathrm{Na}$ Tabela 3 podemos observar o resumo dos histogramas apresentados na Figura 4, resultados obtidos a partir das contagens das nanopartículas das imagens de MET. 
Tabela 3: Diâmetros determinados por MET e seus respectivos desvios padrão.

\begin{tabular}{c|c|c|c|c}
\hline \multirow{2}{*}{ AMOSTRA } & \multicolumn{4}{|c}{ DIÂMETRO } \\
\cline { 2 - 5 } & NP SEM ÓLEO & $\boldsymbol{\sigma}$ & NP COM ÓLEO & $\boldsymbol{\sigma}$ \\
\hline PEO $_{5 \mathrm{kDa}}-b-\mathrm{PLA}_{4,5 \mathrm{kDa}}$ & $38,00 \mathrm{~nm}$ & 1,0 & $58,00 \mathrm{~nm}$ & 2,8 \\
\hline $\mathrm{PEO}_{5 \mathrm{kDa}}-b-\mathrm{PLA}_{10 \mathrm{kDa}}$ & $14,00 \mathrm{~nm}$ & 0,3 & $20,00 \mathrm{~nm}$ & 0,4 \\
\hline
\end{tabular}

Pelos resultados apresentados, podemos observar aumento no tamanho das nanopartículas com a presença do óleo essencial.

Ainda que seja identificada uma certa similaridade entre os diâmetros, é observada uma redução do tamanho das nanopartículas avaliados por MET, comparativamente a técnica de DLS.
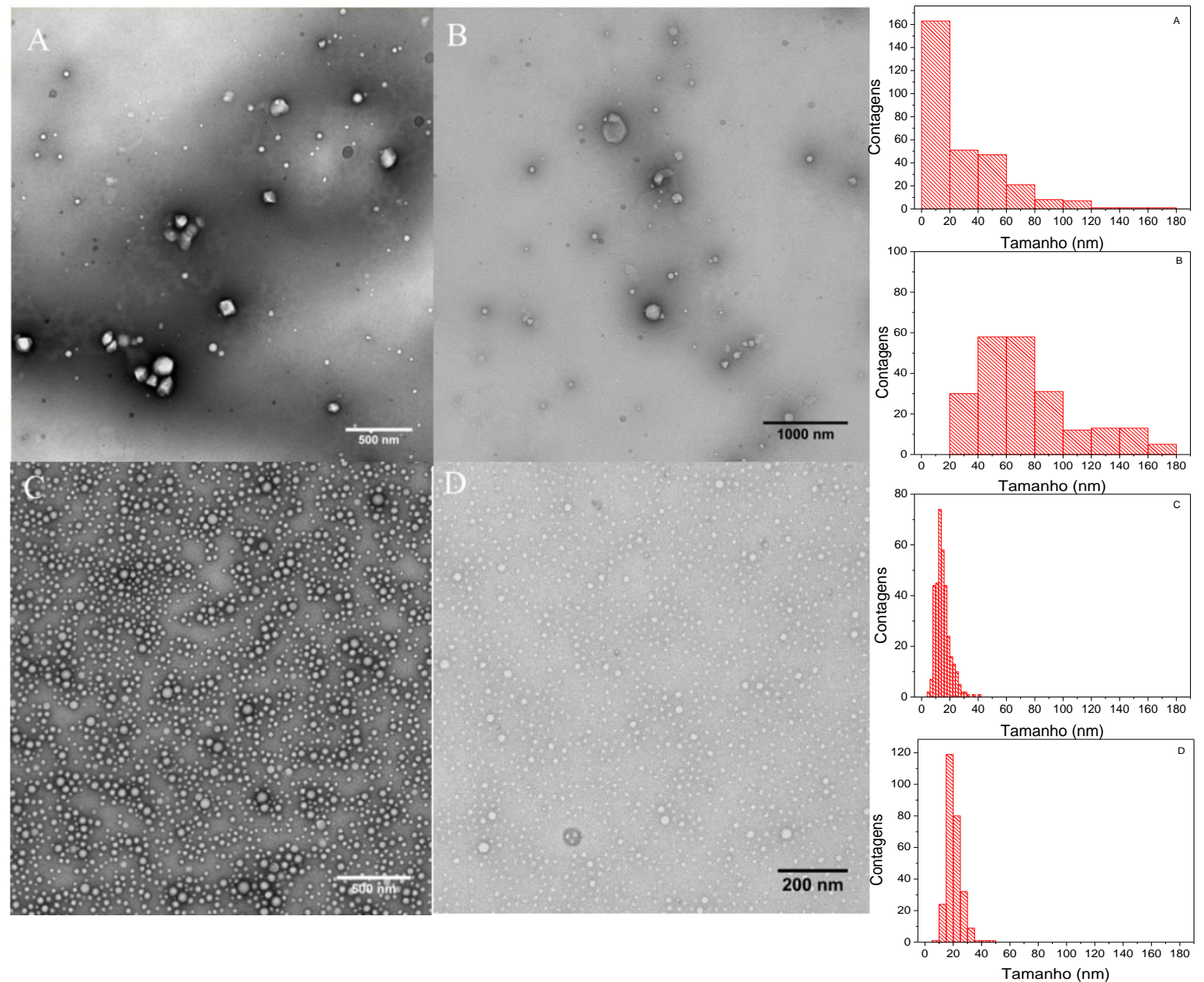

Figura 4: Imagens de MET para as amostras: $\mathrm{PEO}_{5 \mathrm{kDa}}-b-\mathrm{PLA}_{4,5 \mathrm{kDa}}(\mathrm{A}) ; \mathrm{PEO}_{5 \mathrm{kDa}}-b-\mathrm{PLA}_{4,5 \mathrm{kDa}}$ com a BD (B); $\mathrm{PEO}_{5 \mathrm{kDa}}-b-$ $\mathrm{PLA}_{10 \mathrm{kDa}}(\mathrm{C})$; $\mathrm{PEO}_{5 \mathrm{kDa}}-b-\mathrm{PLA}_{10 \mathrm{kDa}}$ com a BD (D). Com os respectivos histogramas das contagens das nanopartículas realizadas pelo software Image Tool.

\subsubsection{Adsorção das nanopartículas em fibras têxteis}

A impregnação das nanopartículas ocorreu por imersão individual de cada amostra em $1 \mathrm{~mL}$ de solução, até total absorção do líquido. Esse valor foi ajustado para um grau de retenção de líquido, relativamente à massa de tecido seco próximo de $100 \%$. Após a impregnação, as amostras foram secas à temperatura ambiente.

Na Figura 5 são apresentadas as imagens digitais (Figuras 5A e 5C) e de microscopia óptica (Figuras 5B e 5D) dos têxteis avaliados.

Os tecidos são divididos em tecidos produzidos em teares e os não tecidos. Os tecidos produzidos em teares geralmente consistem de dois conjuntos de fios, os quais são entrelaçados perpendicularmente uns em 
relação aos outros. [36] O tecido de tear mais utilizado é o tecido plano, definido neste trabalho como tecido A.

A diferença entre os tecidos planos e os não tecidos encontra-se basicamente na forma em que sua estrutura é produzida, [37] como pode se observar na Figura 5. Segundo a NBR 13370, [38] um não tecido é definido como uma estrutura plana, flexível e porosa constituída de véu ou manta de fibras ou filamentos, orientados direcionalmente ou aleatoriamente, e conformados por processo químico, mecânico e/ou térmico, ou pelas combinações desses. Definido neste trabalho como Tecido B. O mesmo processo de impregnação foi aplicado para ambos os materiais.

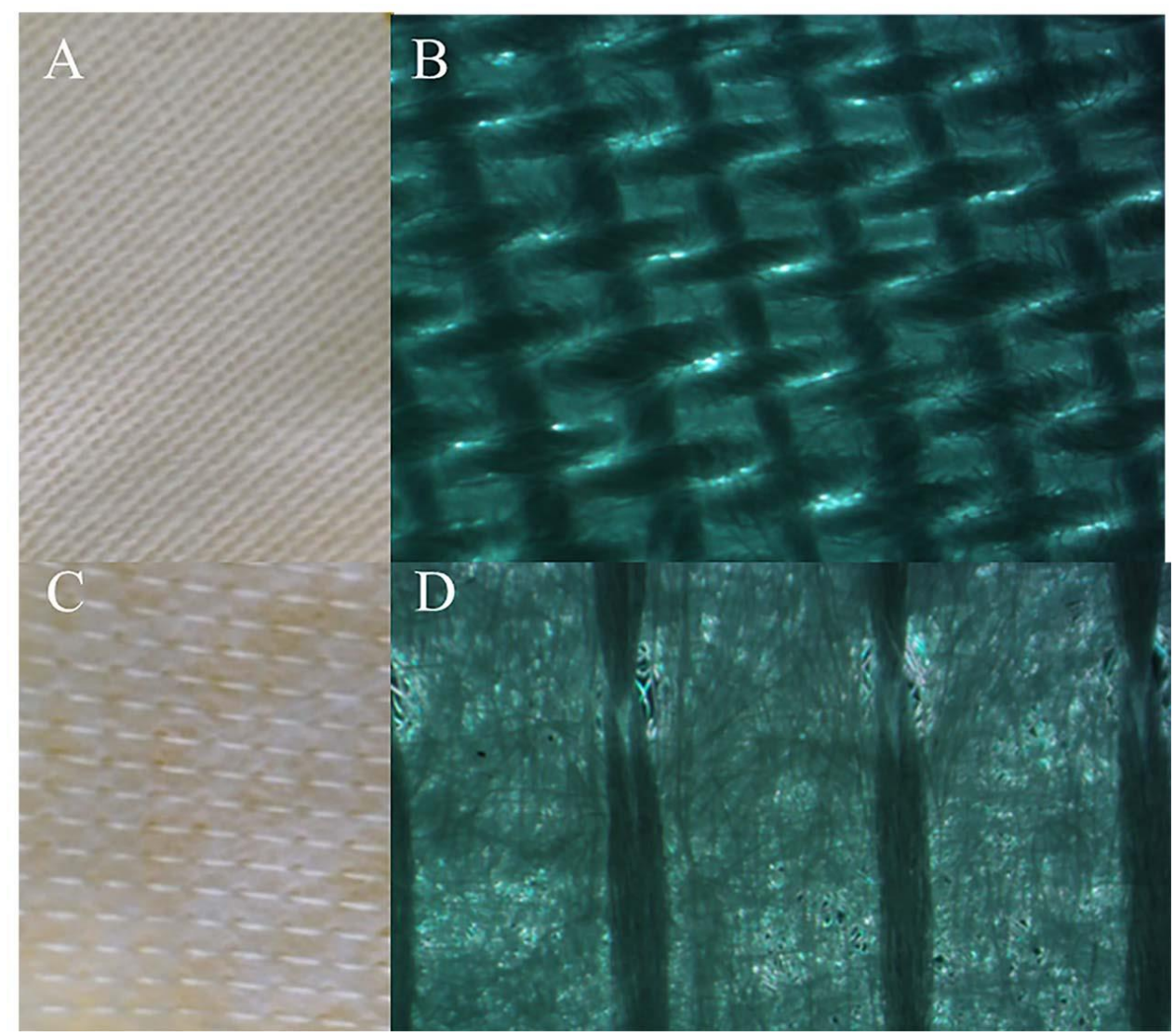

Figura 5: a) Fotografia digital do tecido A (100\% algodão); b) imagem de MO (0,75 de magnificação) do tecido A; c) e d): Fotografia digital e imagem de MO (0,75 de magnificação), respectivamente, do tecido B (100\% poliéster).

\subsubsection{Liberação in vitro}

A técnica de espectroscopia de UV-Visível foi utilizada para confirmar e avaliar as bandas de absorção características do agente ativo e desta forma, determinar a quantidade de óleo essencial liberada. A liberação do agente ativo foi avaliada durante 60 dias e durante este tempo, as amostras foram mantidas em estufa com a temperatura controlada de $37^{\circ} \mathrm{C}\left( \pm 2{ }^{\circ} \mathrm{C}\right)$.

Os resultados mostrados na Figura 6 sugerem que a liberação do óleo essencial ocorre de maneira lenta e controlada. 

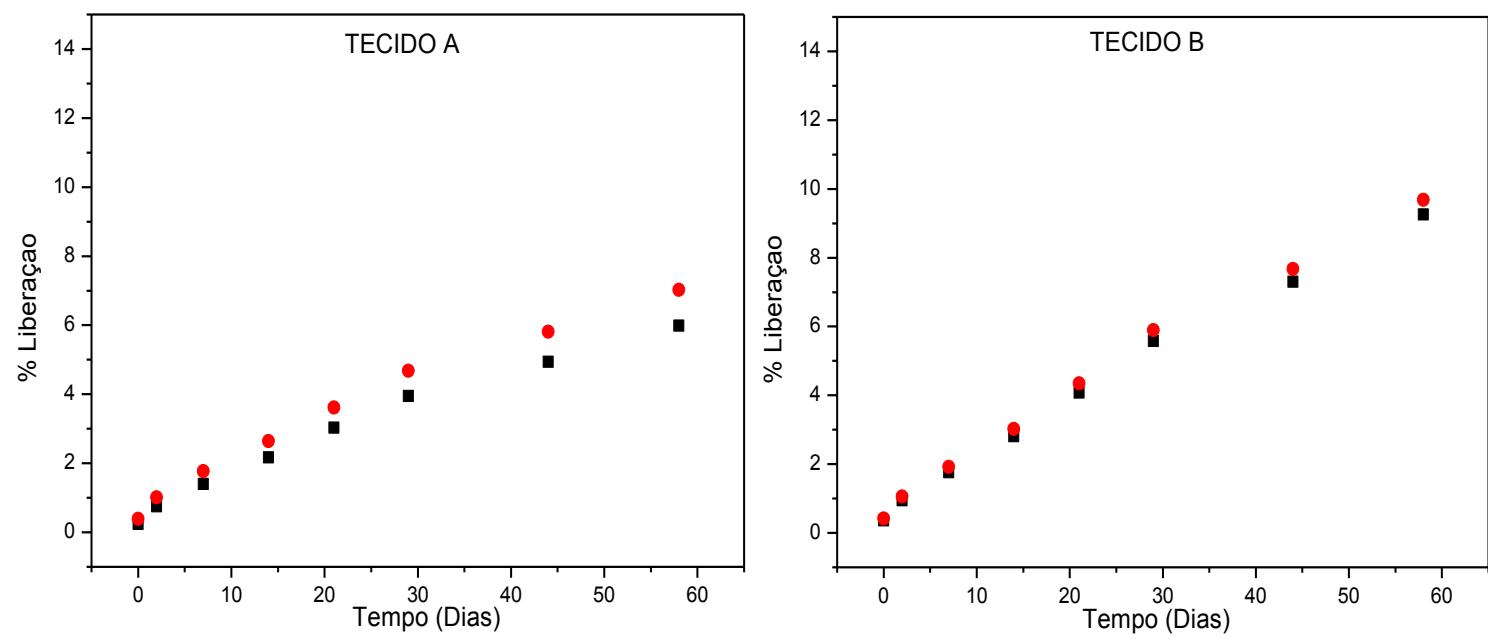

Figura 6: Perfil de liberação do agente ativo das nanopartículas: (O)PEO $\mathrm{PkDa}^{-} b-\mathrm{PLA}_{4,5 \mathrm{kDa}} ;(\boldsymbol{\square}) \mathrm{PEO}_{5 \mathrm{kDa}}-b-\mathrm{PLA}_{10 \mathrm{kDa}}$

As nanopartículas obtidas a partir do copolímero de menor massa, o $\mathrm{PEO}_{5 \mathrm{kDa}}-b$-PLA $4,5 \mathrm{kDa}$ apresentaram uma liberação levemente maior que em comparação ao copolímero $\mathrm{PEO}_{5 \mathrm{kDa}}-b-\mathrm{PLA}_{10 \mathrm{kDa}}$, cerca de $7 \mathrm{e}$ $10 \%$, respectivamente.

Quando comparamos apenas os tecidos, observa-se que no tecido B, a liberação foi levemente maior em relação ao tecido A. Como explicado anteriormente, o tecido A é constituído por fios entrelaçados perpendicularmente uns em relação aos outros, já o tecido B é constituído de uma manta de fibras, dispostos aleatoriamente e esta diferença, embora pequena, foi significativa no efeito antibacteriano discutido a seguir. O perfil de liberação in vitro avaliado em solução das mesmas nanopartículas adsorvidas nas fibras têxteis, sugere que a liberação foi de aproximadamente $10 \%$ para um período de $24 \mathrm{~h}$, caracterizando-se como um processo lento e controlado pelo processo de adsorção das nanopartículas nas fibras têxteis que retardou a liberação do agente ativo.

\subsubsection{Atividade antibacteriana}

A avaliação da atividade antibacteriana foi realizada conforme procedimento descrito na norma técnica $A A$ TCC Test Method 100-2004. Os tecidos A (100\% algodão) e B (100\% poliéster) impregnados por processo de imersão com as nanopartículas de $\mathrm{PEO}_{5 \mathrm{kDa}}-b-\mathrm{PLA}_{4,5 \mathrm{kDa}}$ e $\mathrm{PEO}_{5 \mathrm{kDa}}-b-\mathrm{PLA}_{10 \mathrm{kDa}}$ contendo a mistura de óleos essenciais, foram mantidos em condições controladas de temperatura e umidade. Nestas condições, os tecidos controle A e B (não tratados) apresentaram crescimento bacteriano de $80 \%$ e 93,7 \%, respectivamente, após 24 horas de incubação. Com a incorporação de nanopartículas contendo a mistura de óleos essenciais, as amostras com o tecido A não apresentaram crescimento e/ou redução do número de bactérias. Ao mesmo tempo, uma razoável performance foi observada para o tecido B que apresentou redução da bactéria gram positiva Staphylococcus aureus de 58 e $14 \%$ com a incorporação de nanopartículas de PEO $_{5 \mathrm{kDa}}-b$-PLA $\mathrm{A}_{4,5 \mathrm{kDa}} \mathrm{e}$ $\mathrm{PEO}_{5 \mathrm{kDa}}-b$-PLA $\mathrm{P}_{10 \mathrm{kDa}}$, respectivamente. O melhor resultado observado no tecido $\mathrm{B}$ pode ser explicado pela disposição aleatória das fibras, sugerindo que as nanopartículas estão mais disponíveis para liberar o agente ativo encapsulado quando em contato com o meio de cultura. Com relação a nanopartícula, aquela com menor massa molar de PLA, foi mais eficiente na redução do número de bactérias (58 \%), provavelmente devido ao menor entrelaçamento da cadeia polimérica e mais rápida degradação favorecendo a liberação do óleo essencial. [39, 40]

Os resultados obtidos podem ser comparados com o trabalho de DURÁN et al. [41] que obtiveram excelentes resultados na atividade bacteriostática de tecidos com impregnação de nanopartículas de prata, contra Staphylococcus aureus, obtendo 99,9\% de redução da contagem de bactérias. [43] Em outro estudo, ZHANG et al. [42] avaliaram a atividade antibacteriana de diferentes óleos e, particularmente o óleo essencial de canela mostrou uma inibição de $79 \%$ para a mesma bactéria gram positiva, Staphylococcus aureus. [44] NAVARRO et al. [43] avaliaram o óleo essencial natural de melaleuca em usuários de calçados, confirmando atividade antibacteriana contra diferentes bactérias encontradas na pele dos pés e em e sapatos desgastados. [45]

Os resultados corroboram com a análise feita por espectroscopia de UV-Visível, pois o tecido B apresentou uma redução da atividade bacteriana e também demonstrou um melhor perfil de liberação do agente ativo, mostrando ser o sistema mais eficiente entre os estudados. As baixas quantidades de aditivo antibacte- 
riano utilizadas para os sistemas estudados, com a obtenção de resultados satisfatórios, garantem também que essas quantidades não afetam as características físicas de cor e textura do material têxtil.

\section{CONCLUSÕES}

As nanopartículas foram obtidas pelo método de nanoprecipitação dos copolímeros $\mathrm{PEO}_{5 \mathrm{kDa}}-b$ - $\mathrm{PLA}_{4,5 \mathrm{kDa}} \mathrm{e}$ $\mathrm{PEO}_{5 \mathrm{kDa}}-b-\mathrm{PLA}_{10 \mathrm{kDa}}$. O raio hidrodinâmico das nanopartículas manteve-se de 14 a $75 \mathrm{~nm}$, e foi observada uma dependência do tamanho do bloco de PLA. Considerando-se que os sistemas nanoestruturados podem encapsular agentes ativos fracamente solúveis em água, tais como óleos essenciais, conclui-se que as nanopartículas obtidas a partir de copolímeros de PEO- $b$-PLA foram eficazes em incorporar a blenda de óleos essenciais utilizada. O efeito antibacteriano da blenda de óleos essenciais utilizada em nanopartículas de PEO- $b$-PLA demonstrou atividade bacteriostática contra Staphylococcus aureus, reduzindo em 58\% o crescimento desta bactéria para nanopartículas de $\mathrm{PEO}_{5 \mathrm{kDa}}-b-\mathrm{PLA}_{4,5 \mathrm{kDa}}$ sugerindo seu potencial uso como agente antibacteriano. A eficiência antibacteriana confirmada pelas análises microbiológicas utilizando metodologias internacionais (AATCC Test Method 100-2004) foi mais efetivo em tecido construído com fibras dispostas aleatoriamente (Tecido B). Corroborando com estudos da literatura, as nanopartículas obtidas a partir de copolímeros com menor massa molar de PLA, ou seja, $\mathrm{PEO}_{5 \mathrm{kDa}}-b-\mathrm{PLA}_{4,5 \mathrm{kDa}}$, favoreceram a liberação do óleo essencial, 7 e $10 \%$ em 60 dias de avaliação, e consequentemente a redução do número de bactérias. O processo de incorporação da blenda de óleos essenciais nas fibras têxteis mostrou-se eficiente no tempo estudado mesmo com ação da temperatura, sugerindo viabilidade quanto ao uso deste sistema no controle antibacteriano. Outro aspecto a considerar é a característica lenta e controlada da liberação do ativo a partir do sistema nanoestruturado avaliado.

\section{AGRADECIMENTOS}

Os autores agradecem o suporte financeiro recebido de CERMAV, CNRS, CAPES, CNPq, Universidade Federal de Santa Catarina (UFSC) e Instituto Brasileiro de Tecnologia do Couro, Calçado e Artefatos (IBTeC). As empresas NanoVetores e Dublauto pelo auxílio com os materiais. Centre de Recherches sur les Macromolécules Végétales (CERMAV-CNRS), Universidade de Grenoble, Grenoble, France, particularmente ao Dr. R. Borsali.

\section{BIBLIOGRAFIA}

[1] PANIZ, O.G., SCHEIK, L.K., SILVA, G.E.H., et al., "Obtenção de compósito com matriz de acetato de celulose e partículas de prata para aplicações antimicrobianas”; Matéria (Rio J.) [online], v.23, n.4, 2018.

[2] GAO, Y., CRANSTON, R., "Recent Advances in Antimicrobial Treatments of Textiles"; Textile Research Journal, v. 78, n. 1, pp. 60-72, Jan. 2008.

[3] GORENSEK, M.; RECELJ, P.; "Nanosilver Functionalized Cotton Fabric"; Textile Research Journal, v. 77, n. 3, pp. 138-141, Mar. 2007.

[4] CZAJKA, R., "Development of medical textiles"; Fibers and Textiles in Eastern Europe, v. 13, pp. 13-15, 2005.

[5] PORTO, R.C.T., UCHÔA, P.Z., PESCHEL, L.T., et al., "Nanopartículas de óxido de zinco sintetizadas pelo método poliol: caracterização e avaliação da atividade antibacteriana"; Matéria (Rio J.) [online], v. 22, s.1, 2017.

[6] BACKX, B.P., DELAZARE, T., "The influence of dispersive medium on efficient and eco-friendly synthesis of silver nanoparticles for application in antimicrobial fabric", Revista Verde, v. 14, n. 2, pp. 252-257. 2019.

[7] GULRAJANI, M.L., GUPTA, D., "Emerging techniques for functional finishing of textiles", Indian Journal of Fibre \& Textile Research. v.36, pp. 388-397, Dec. 2011.

[8] BALASUBRAMANIAN, K., KODAM, K.M., "Encapsulation of therapeutic lavender oil in an electrolyte assisted polyacrylonitrile nanofibres for antibacterial applications"; RSC Advances, v. 4, pp. 5489254901, Sep. 2014.

[9] JU, A., http://www.news.cornell.edu/stories/May07/nanofibers.fashion.aj.html, acessada em Julho 2012.

[10] CORBIERRE, M.K.; CAMERON, N.S.; SUTTON, M., et al., "Polymer-stabilized gold nanoparticles and their incorporation into polymer matrices"; Journal of the American Chemical Society, v. 123, n. 42, pp. 10411-2, Oct. 2001.

[11] CORBIERRE, M.K.; CAMERON, N.S.; SUTTON, M., et al., "gold nanoparticle/polymer nanocompo- 
sites: dispersion of nanoparticles as a function of capping agent molecular weight and grafting density"; langmuir, v. 21, n. 13, pp. 6063-72, jun. 2005.

[12] NAM, S., PARIKH, D.V., CONDON, B.D., et al., "Importance of poly(ethylene glycol) conformation for the synthesis of silver nanoparticles in aqueous solution"; Journal of Nanoparticle Research, v. 13, n. 9, pp. 3755-3764, Sep. 2011.

[13] BALAKUMARAN, M.D., RAMACHANDRAN, R., JAGADEESWARI, S., et al., "In vitro biological properties and characterization of nanosilver coated cotton fabrics - An application for antimicrobial textile finishing” International Biodeterioration \& Biodegradation, v. 107, pp. 48-55, Feb. 2016.

[14] REINSTE, http://www.reinste.com/index.html, acessada em Agosto 2012.

[15] http://polymer-additives.specialchem.com/news/industry-news/reinste-signs-agreement-with-germanybased-silanotex-for-nano-silver-masterbatches, acessada em Agosto 2012.

[16] JONES R., http://www.complex.com/sneakers/2013/10/know-your-tech-reebok-playdry, acessada em Novembro 2016.

[17] http://www.mundodastribos.com/sapato-social-masculino-ferracini.html, acessada em Novembro 2016.

[18] Revista Inovação - Nov/dez 2010 e jan. 2011.

[19] http://www.nanovetores.com.br/, acessada em Abril 2015.

[20] Têxteis com proteção microbiana, Citeve, 2008.

[21] http://tnsolution.com.br/2015/08/12/antimicrobianos-quais-as-diferencas//, acessada em Novembro 2016.

[22] POPIOLSKI, T.M., OTSUKA, I., HALILA, S., et al., "Preparation of polymeric micelles of poly(ethylene oxide-b-lactic acid) and their encapsulation with lavender oil"; Materials Research. (Sao Carlos, Braz.), v. 19, n. 6, Sep. 2016.

[23] PORTO, L. C., Estudo de filmes finos e de nanopartículas obtidos pela auto-associação de copolímeros em bloco e sua interação com um oligo/polissacarídeo; Tese de doutorado; UFSC, Florianópolis, SC, Brasil, 2011.

[24] ZHANG, Q., LIN, J., WANG, L., et al., "Theoretical modeling and simulations of self-assembly of copolymersin solution"; Progress in Polymer Science, v. 75, pp. 1-30, Dec. 2017.

[25] SCHÄRTL, W., Light scattering from polymer solutions and nanoparticle dispersions; Germany, Springer Laboratory, 2007.

[26] PROVENCHER, S.W., "Inverse problems in polymer characterization: Direct analysis of polydispersity with photon correlation spectroscopy"; Makromolekulare Chemie, v.180, pp. 201-209, 1979.

[27] PROVENCHER, S.W., "An eigenfunction expansion method for the analysis of exponential decay curves"; Journal of Chemical Physics, v. 64, n. 7, pp. 2772-2777, Ap. 1976.

[28] DAL BÓ, A.G., SOLDI, V., GIACOMELLI, F.C., et al., "Self-assembly of amphiphilic glycoconjugates into lectin-adhesive nanoparticles"; Langmuir, v. 28, n. 2, pp. 1418-1426, Jan. 2012.

[29] SIRVAITYTE, J., SIUGZDAITE, J., VALEIKA, V., "Application of commercial essential oils of eucalyptus and lavender as natural preservative for leather tanning industry"; Chemical Reviewv, v. 62, n. 9, pp. 884-893, Sep. 2011.

[30] TUREK, C., STINTZING, F.C., "Application of high-performance liquid chromatography diode array detection and mass spectrometry to the analysis of characteristic compounds in various essential oils"; Analytical and Bioanalytical Chemistry, v. 400, n. 9, pp. 3109-3123, Jul. 2011.

[31] AATCC - American Association of Textile Chemists and Colorists, Test Method for Antimicrobial Finishes 100-2004.

[32] SCHAFFAZICK, S.R., POHLMANN, A.R., DE LUCCA FREITAS, L., et al., "Caracterização e estudo de estabilidade de suspensões de nanopartículas e de nanoesferas poliméricas contendo diclofenaco"; Acta Farmaceutica Bonaerense, v. 21, n. 2, pp. 99-106, Feb. 2002.

[33] DUMITRIU, S., Polysaccharides: Structural diversity and functional versatility; Burchard, $2^{\mathrm{a}}$ Ed, cap 7, Marcel Dekker, Inc., Nova York, 1998.

[34] XU, R., WINNIK, M.A., "Light-scattering study of the association behavior of styrene-ethylene oxide block copolymers in aqueous solution"; Macromolecules, v. 24, n. 1, pp. 87-93, Jan. 1991.

[35] REIS, C.P., NEUFELD, R.J., RIBEIRO, A.J., et al., "Nanoencaps $\mu$ lation I. Methods for preparation of drug-loaded polymeric nanoparticles"; Nanomedicine: Nanotechnology, Biology, and Medicine, v. 2, n. 1, pp. 
8-21, Mar. 2006

[36] JIE, P., VENKATRAMAN, S.S., MIN, F., et al., "Micelle-like nanoparticles of star-branched PEO-PLA copolymers as chemotherapeutic carrier"; Journal of Controlled Release, v. 110, n. 1, pp. 20-33, Dec. 2005.

[37] NECKEL, G.L., SENNA, E.L., "Preparação e caracterização de nanopartículas contendo camptotecina a partir do ácido poli(D,L-lático) e de copolímeros diblocos do ácido poli(D,L-lático) e polietilenoglicol”; Acta Farmaceutica Bonaerense, v. 24, n. 4, pp. 504-11, Jul. 2005.

[38] ARAÚJO, M., CASTRO, E.M.M., Manual de engenharia têxtil; Portugal, Lisboa: Fundação Calouste Gulbenkian, 1984.

[39] FEITOR, M.C., Efeito antibacteriano de tecidos têxteis revestidos por prata através da técnica de deposição por plasma; Tese de doutorado; UFRN, Natal, RN, Brasil, 2010.

[40] NBR 13370: 2002, Não tecido - Terminologia, ABNT - Associação Brasileira de Normas Técnicas.

[41] FENG, S., NIE, L., ZOU, P., et al., "Effects of drug and polymer molecular weight on drug release from PLGA-mPEG microspheres" Journal of Applied Polymer Science, v. 132, n. 6, Aug. 2015.

[42] LIU, R., WANG, Y., MA, Y., et al., "Effects of the molecular weight of PLGA on degradation and drug release in vitro from an mPEG-PLGA nanocarrier" Chemical Research in Chinese Universities, v. 32, n. 5, pp. 848-853, Aug. 2016.

[43] DURÁN, N., MARCATO, P.D., SOUZA, G.I.H. et al., "Antibacterial effect of silver nanoparticles produced by fungal process on textile fabrics and their effluent treatment"; Journal of Biomedical Nanotechnology, v. 3, n. 2, pp. 203-208, Jun. 2007.

[44] ZHANG, Y., LIU, X., WANG, Y., et al., "Antibacterial activity and mechanism of cinnamon essential oil against Escherichia coli and Staphylococcus aureus"; Food Control. v. 59, pp. 282-289, Jan. 2016.

[45] NAVARRO, M.M.S., GARROTE, N.C., AÍS, F.A., et al., "Microencapsulation of melaleuca alternifolia (tea tree) oil as biocide for footwear applications"; Journal of Dispersion Science and Technology, v. 32, pp. 1722-1727, Nov. 2011.

\section{ORCID}

Tatiane Michele Popiolski

https://orcid.org/0000-0002-7954-0901

Markus Wilimzig

https://orcid.org/0000-0003-4587-9736

Valdir Soldi

https://orcid.org/0000-0002-4316-3622 